Western University

Scholarship@Western

2015

Dye Distance Mapping using Waveguide Evanescent Field Fluorescence Microscopy and its Application to Cell Biology

Frederik Fleissner

Western University

Michael Morawitz

Western University

S. Jeffrey Dixon

Western University

Uwe Langbein

Western University

Silvia Mittler

Western University, smittler@uwo.ca

Follow this and additional works at: https://ir.lib.uwo.ca/physicspub

Part of the Astrophysics and Astronomy Commons, and the Physics Commons

Citation of this paper:

Fleissner, Frederik; Morawitz, Michael; Dixon, S. Jeffrey; Langbein, Uwe; and Mittler, Silvia, "Dye Distance Mapping using Waveguide Evanescent Field Fluorescence Microscopy and its Application to Cell Biology" (2015). Physics and Astronomy Publications. 39.

https://ir.lib.uwo.ca/physicspub/39 
Journal of Biophotonics 8 (2015) 826-837

\title{
Dye Distance Mapping using Waveguide Evanescent Field Fluorescence Microscopy and its Application to Cell Biology
}

\author{
Frederik Fleissner ${ }^{1,2}$, Michael Morawitz ${ }^{1,2}$, S. Jeffrey Dixon ${ }^{3}$, Uwe Langbein ${ }^{2}$ \\ and Silvia Mittler ${ }^{*}$ \\ 1Department of Physics and Astronomy, The University of Western Ontario (Western University), London, Ontario, \\ N6A 3K7, Canada \\ ${ }^{2}$ RheinMain University of Applied Sciences, Department of Engineering Sciences/ \\ Institute of Microtechnologies, Am Brückweg 26, 65428 Rüsselsheim, Germany \\ ${ }^{3}$ Department of Physiology and Pharmacology, Schulich School of Medicine \& Dentistry, The University of \\ Western Ontario (Western University), London, Ontario, N6A 5C1, Canada
}

\begin{abstract}
Previous studies have measured the distance between cells and the substratum at sites of adhesion via the emission of a fluorescent dye and waveguide methods. Here, we demonstrate a novel approach to measure the position of fluorescent dyes above a waveguide surface in the 10-200 nm distance range throughout an entire area, yielding a 2D dye distance map or a 3D contour plot. The dye is located in a multilayered Langmuir Blodgett (LB) film or in the plasma membrane of a cell. Waveguide evanescent field fluorescence (WEFF) images obtained using two different waveguide modes are employed allowing, with a simple mathematical approach, the calculation of dye distance maps. Ultra-thin steps made using LB technology, adhesion distances and the bending of the plasma membrane between focal adhesions of osteoblastic cells are shown as examples. The errors are discussed.
\end{abstract}

\footnotetext{
*To whom correspondence should be addressed:.E-mail:smittler@uwo.ca;
} Phone: ++ 1 (519) 661-2111 loc.88592; Fax: ++ 1 (519)661-2033

Key words: Waveguide Evanescent Field Fluorescence (WEFF) Microscopy, optical waveguide, dye location from waveguide surface, LB films, osteoblasts, cell-substrate contacts 


\section{Introduction}

With the aim of developing new medical devices with direct tissue contact, drug delivery vehicles, and tissue engineering scaffolds, there has been increasing interest in recent years in the interactions of cells with both synthetic and natural biomaterials [1,2]. In particular, the study of the contact regions between a cell and its substratum is of considerable interest as its investigation delivers inter alia information about the cytocompatibility of the substratum - the affinity of cells towards that particular surface. Promotion or inhibition of cell adhesion to synthetic and natural biomaterials is often crucial to the proper function of a particular device. Some information concerning these interactions, e.g. the lateral location and the density of the adhesion sites, as well as their relationship to the actin stress fiber system, part of the cell's cytoskeleton, can be inferred from fluorescence microscopy of immunolabeled molecules involved in adhesion; typically, vinculin, a protein located within the multi-protein complex that anchors the adhesion to the cytoskeleton inside the cell [3]. These methods only deliver signals from the focus volume and no information about adhesion distances to the substratum. However, a direct and quantitative method to address the distance to the substratum is highly attractive. To address this need, different microscopic techniques based on electron microscopy [4] and optical means such as evanescent fields and interference techniques have been developed. Total internal reflection fluorescence (TIRF) [3,5], surface plasmon resonance microscopy (SPRM) [6], interference fluorescence microscopy (IRM) [7], fluorescence interference contrast (FLIC) microscopy [8] and combinations thereof $[3,9]$ have been used to visualize and quantify these contacts. The contacts themselves had been discovered by interference reflection microscopy (IRM) in the 1970s [10]. 
Recently, waveguide evanescent field fluorescence (WEFF) microscopy was developed with the ability to image ultra-thin, solid thin films and cell-substratum contact regions of cells [11-16]. Dynamic and static investigations of cell-substratum contacts have been performed [1416] as well as multimode waveguide analysis to estimate separation distances at particular locations [15].

These methods allow one to distinguish between the different kinds of integrin-mediated adhesions present in cells via their specific separation distances from the substratum. These contacts are called integrin-mediated as the "physical" contact between the cell and proteins coating the substratum is accomplished by transmembrane proteins known as integrins. Focal adhesions (FAs) show separation distances of 10-20 nm between the substratum and the ventral (front or outer) side of the plasma membrane, with a cytoplasmic, multi-protein anchor complex extending up to $60 \mathrm{~nm}$ into the cell from the membrane. FAs are often found close to the leading edge of a cell in early culture. Close contacts (CCs) are defined with separation distances of 30-50 $\mathrm{nm}$ and often occupy larger surface areas. Extracellular matrix contacts (EMCs) show the largest separation distances of $>50 \mathrm{~nm}$ and are typically found in enhanced numbers in later cultures [4]. However, separation distances of point contacts (PCs), which are found at the tips of filopodia and the periphery of cell bodies, but show slightly different multi-protein anchor complexes than FAs and involve different integrins $[17,18]$, have not been described so far. FA, CC, EMC and PC involve integrins (but not necessarily the same) as transmembrane proteins, which serve as the physical link between the substratum and the multi-protein anchor complex in the cytoplasm. This protein anchor complex is connected via actin stress fibers to the overall cytoskeleton and therefore dictates the form or habitus of the cell [19]. 
In WEFF microscopy, slab waveguides are implemented as both microscopy substrata and illumination sources. The generation of an evanescent field above the waveguide - in this work of up to $\sim 250 \mathrm{~nm}$ in height but depending on the individual waveguide - allows the excitation of fluorescent dyes located in this "thin film of light" on the waveguide. In the case of an adherent cell, this illuminated volume covers mainly the adhesions, whereas the remaining cell body is not irradiated. Therefore, only stained regions close to the waveguide surface contribute to the generated fluorescence image. With respect to illumination technology, this is similar to TIRF microscopy with fixed TIR angles. Figure 1 depicts a general scheme of the method. Overexposure in WEFF microscopy leads to images which are very similar to bright field images. Scattered light, always present in a waveguide system, leads to 3D illumination and 3D emission of fluorescence creating an epi-fluorescence image.

Up until now, distances between fluorescent probes and the substratum have only been estimated with the help of WEFF microscopy on individual adhesion points on cells taking a table into account which was based on calculations of differences in individual mode intensities at different positions in the evanescent field [15]. Here we describe a novel method to map the dye location above a waveguide surface in a $2 \mathrm{D}$ fashion by implementing a very simple mathematical treatment involving two well characterized propagating waveguide modes and the simulation of the evanescent field under experimental conditions. This allows for the determination of the location of dye molecules responsible for the image and its contrast, delivering information throughout an entire area and not at singular points only. The mathematical concept was integrated into in-house written image analysis software. Two kinds of well-characterized samples were investigated: a stepped multilayer LB film with known step heights and fixed osteoblasts as adhesion data from these cells are known [15,20-22]. 


\section{Experimental Methods}

\section{Waveguide evanescent field fluorescence (WEFF) microscopy}

The WEFF microscope [14] consisted of an inverted microscope (Carl Zeiss, Oberkochen, Germany) with the waveguide placed on the sample stage. The specimen was located on top of the waveguide. An argon ion laser (35 LAP 341-200, CVI Melles Griot) operated at $\lambda=488 \mathrm{~nm}$ with a variable output power of 7- $126 \mathrm{~mW}$ was used as the light source. A neutral density filter was placed directly behind the laser for power reduction, avoiding bleaching and overexposure. An iris aperture controlled the beam diameter. The laser beam was coupled into a chosen waveguide mode by a coupling grating located on the waveguide. To block the undesired excitation wavelength, a long pass filter with a cut-off wavelength of $\lambda_{\text {cut-off }}=490 \mathrm{~nm}$ (3RD490LP, Omega Optics, Brattleboro, VT) was fitted between the objective and the camera. In this upgraded set-up, the outcoupled intensity at the end of the waveguide was captured with a large active area photodiode (FDS1010, Thorlabs, Newton, NY). With the help of this signal, a relative coupling efficiency could be determined for each coupled mode used in image acquisition. A cooled CCD-camera (Persuit-XS 1.4 Diagnostic Instruments Inc., Sterling Heights, MI), controlled with SPOT 5 Basic (Spot Image Solutions, Sterling Heights, MI) was connected to a computer. Image data were exported for processing. Additionally, bright field microscopy images of the samples were captured with the same field of view/objective lens as the WEFF microscopy images and processed with Image Pro Express (Media Cybernetics, Rockville, MD). All images which were used for distance mapping had an exposure time of $180 \mathrm{~s}$. It was recently proven that this exposure time does not lead to photo bleaching issues [23]. 


\section{Waveguide fabrication and characterization}

Step-index waveguides were fabricated with a home built RF-sputtering system based on a Leybold Heraeus vacuum chamber (030 16 Br. 1; Oerlikon Leybold Vacuum GmbH, Cologne, Germany) and a Hüttinger PFG 1000 RF-system (Hüttinger Elektronik, Freiburg, Germany). The sputtered waveguide material was P-LASF 47 glass (nP-LASF 47 at $\lambda=488 \mathrm{~nm}$ : 1.81994; Schott, Mainz, Germany) onto fused silica substrates ( $\mathrm{n}_{\text {fused silica }}$ at $\lambda=488 \mathrm{~nm}$ : 1.46302; FQ VIS2 Hebo, Aalen, Germany). Before sputtering the guiding layer, a waveguide mode coupling grating was fabricated by a laser interference holography set-up in photoresist $[24,25]$ and then milled into the fused silica substrate via reactive ion etching (Vacu Tec Plasma Systems, control unit: CPU 500, RF-Generator: ENI ACG-3 XL, Matching-Box: Vacu Tec Plasmatch, Gas/Flow-Meter: VFC 500) with sulfur hexafluoride. Multimode waveguides with a thickness of $\sim 650 \mathrm{~nm}$, enabling three modes in each polarization direction at $\lambda=488 \mathrm{~nm}$, were fabricated. The coupling gratings had periodicities $\Lambda$ in the order of $610 \pm 3 \mathrm{~nm}$.

These waveguides were characterized by a standard waveguide characterization set-up [26] to determining waveguide refractive index and thickness. With these data, the field distributions of all modes were calculated with ATSOS (Analysis Tool for Stratified Optical Structures) [27], delivering the necessary information about the evanescent field in the cladding. For these simulations the cladding refractive index, $n_{1}$ was chosen according to the sample: in the case of the stearic acid LB-films, $n_{1, s a}=1.47$ at $\lambda=588 \mathrm{~nm}[28]$ and, in the case of fixed cells, $n_{1, w a t e r}=$ 1.33 for water was used. A simulation example can be found in the Supplemental Material for $\lambda=$ $488 \mathrm{~nm}$, a substrate with $n=1.460$ and a waveguide with $658 \mathrm{~nm}$ thickness and a refractive index of $n=1.841$ with water as cover medium at $n=1.33$. 


\section{Waveguide cleaning}

Before LB-deposition or cell plating onto the waveguides substrata, they had to be cleaned to remove any contaminants and to provide a chemically homogeneous surface. The substrata were first cleaned with compressed air and then rinsed with acetone, deionized water and isopropanol. Depending on the previous use of the waveguide, it was either immersed for 5 minutes in chloroform or for 15 minutes in ethanol. To remove organic material, the dried samples were cleaned with Nano-Strip (KMG Chemicals Inc., Fremont, CA) at $80^{\circ} \mathrm{C}$ for 5 minutes. After the Nano-Strip application, the substrata were rinsed extensively in Milli-Q water. For further purification and to make the glass surface hydrophilic, the substrates were treated with an oxygenplasma (STS, 300 Series, Northern Telecom) for $30 \mathrm{~s}$.

\section{Langmuir-Blodgett films}

The Langmuir-Blodgett method [29] was applied to fabricate a sample with a controlled dye-waveguide-surface distance (Fig.2). Fluorescence-inactive layers of stearic acid (p.a. grade, Fluka) were prepared from a solution with a concentration of $1 \mathrm{mg} / \mathrm{ml}$ in chloroform. An actively fluorescing layer was fabricated from a mixture of the dye DiO (3,3'-dioctadecyloxacarbocyanine perchlorate: $\mathrm{C}_{53} \mathrm{H}_{85} \mathrm{ClN}_{2} \mathrm{O}_{6}$, Biotium, Hayward, CA) and DPPC (1, 2-dipalmitoyl-sn-glycero-3phosphatidylcholine, Sigma Aldrich). DiO is a typical lipophilic tracer dye used to stain membranes. DPPC is necessary to dilute the dye molecules in the monolayer. Undiluted DiO is quenched and does not fluoresce. Both individual materials were dissolved in chloroform at 1 $\mathrm{mg} / \mathrm{ml}$ and a mixture of 25/1 vol/vol DPPC/DiO was prepared. The stearic acid was deposited at a surface pressure of $35 \mathrm{mN} / \mathrm{m}$ as 3, 13 and 23 layers (Fig.1) in a staircase fashion. The dye mixture (DPPC/DiO) was deposited as a monolayer on top of the entire stearic acid step sample at a surface 
pressure of $30 \mathrm{mN} / \mathrm{m}$. All preparation steps were carried out on a KSV 3000 trough (KSV Nima, Espoo, Finland) in a clean room environment at room temperature. After droplet application to the air-water interface, solvent was allowed to evaporate for 20 minutes before films were compressed. The subphase was a solution of $1 \mathrm{~g} / 1 \mathrm{MnCl}_{2}$ (99.99\%, Sigma Aldrich) in Milli-Q water (Millipore, Billerica, MA). The film lift was operated at $2 \mathrm{~mm} / \mathrm{s}$. Transfer ratios decreased from $\sim 1$ to 0.86 during multilayer deposition. The LB film samples were characterized by scanning electron microscopy (SEM: LEO 1540XB, Zeiss, Oberkochen, Germany).

\section{Cell culture, fixation and labelling}

The osteoblastic cell line MC3T3-E1 (subclone 4, ATTC Catalog 3 CRL-2593) were cultured in flasks. The cleaned waveguides were sterilized for 3 hours by UV light. Growth medium was prepared from $17.8 \mathrm{ml} \alpha$-minimum essential medium 1X (MEM; Gibco), $2 \mathrm{ml}$ fetal bovine serum (FBS; Gibco) and $0.2 \mathrm{ml}$ antibiotic-antimycotic solution 100X (Anti-Anti; Gibco). First the medium was aspirated from the cell culture flask. Dulbecco's phosphate-buffered saline 1X (PBS; Gibco) was added to wash the cell layer and aspirated subsequently. To detach the osteoblasts from the vessel wall, $5 \mathrm{ml}$ trypsin-EDTA ( $0.05 \%$, Gibco) was added and incubated at $37^{\circ} \mathrm{C}$ for 5 minutes. The culture was checked by phase-contrast microscopy to confirm that cells were released into the suspension. The trypsin was neutralized by adding $9 \mathrm{ml}$ growth medium to the flask. The resulting cell suspension was diluted in growth medium to 10,000 cells per ml. Waveguides were placed in a Petri dish and $1 \mathrm{ml}$ cell suspension per substrate was applied to the surfaces. Samples were then incubated for $24 \mathrm{~h}$ at $37^{\circ} \mathrm{C}, 100 \%$ humidity and $5 \% \mathrm{CO}_{2}$.

The waveguides were removed from the growth medium and excess medium was aspirated. Next, each waveguide was rinsed three times in PBS. For fixation, the waveguides with the cells 
on top were placed in a solution of $4 \%$ paraformaldehyde in PBS for 10 minutes at room temperature. Subsequently, samples were rinsed three times with PBS. To prevent desiccation, samples were kept in PBS until further treatment. A solution was prepared from $1.5 \mathrm{mg} \mathrm{DiO}$ in 1 $\mathrm{ml}$ dimethyl sulfoxide (DMSO) and heated to $37^{\circ} \mathrm{C}$ within 5 minutes. This mixture was sedimented for 5 minutes at $2000 \mathrm{rpm}$ to separate solid residues. Ten $\mu \mathrm{l}$ of this stock solution was dissolved in $1 \mathrm{ml}$ growth medium to form the staining solution. The staining solution $(200 \mu \mathrm{l})$ was pipetted onto the corner of each waveguide and the waveguide gently agitated until all cells were covered with staining solution. The samples were left in the solution for 20 minutes to incorporate the dye. Afterwards, the staining solution was drained and the waveguides were washed in PBS. For the removal of all unbound dye, the samples were immersed in PBS for 10 minutes and drained again. The entire wash cycle was repeated two more times. The waveguides were stored in PBS until performing WEFF microscopy. This procedure delivers fixed cells, cells that are "frozen" in their habitus [30-32] with the dye situated in the plasma membrane of the cells.

\section{Image acquisition, relative coupling efficiency determination and normalization}

For each distance map, $x(y ; z)$, two grey scale images (1024 x 1360 pixel, 14 bit) were taken with two different waveguide modes, $m_{i}$, however identical exposure times. For further image processing, both images were converted to matrices, $\mathrm{G}_{\mathrm{i}}(\mathrm{y} ; \mathrm{z})$ where the values represent pixel intensities. For each waveguide mode, a relative coupling efficiency was measured by the photodiode at the end of the waveguide, $V_{i, P D}$. In addition, an overexposed image (similar to epifluorescence image) was taken at the identical magnification and field of view. Background noise from ambient light and CCD bias were subtracted from the acquired images. 
Normalization of the images with respect to the coupling efficiency was necessary as the theoretical framework (ATSOS) for calculating the field distribution in and on the waveguide (after waveguide characterization) assumes that both modes carry the same total power flow. In reality, the guided power depends on the properties of the coupling grating and differs for each individual measurement, being a function of alignment. We anticipated and have proven [14] that the overall intensity in the image is proportional to the intensity in the evanescent field and that this is proportional to the guided intensity and therefore proportional to the intensity leaving the waveguide at its end; the signal of the photodiode. Therefore, both image matrices, $G_{i}(y ; z)$ were

normalized by the relative coupled intensities, $I_{i, P D}$. The normalized image matrices $G_{i, n o r m}(y ; z)$ are then given by

$$
G_{i, n o r m}(y ; z)=\frac{G_{i}(y ; z)}{I_{i, P D}}
$$

\section{Mathematical formalism for distance determination}

A theoretical model was created based on the fact that the brightness of a fluorescence signal in the microscopy image from a dye in an evanescent field is related to a) the distance from the waveguide surface and, therefore, $b$ ) the particular waveguide mode by which the evanescent field is created (Fig.3). This assumes that dye molecules are not distributed in the distance direction, $\mathrm{x}$, but located at one position only, a reasonable assumption for both the LB film steps and stained plasma membranes of cells.

Each individual waveguide mode caused an evanescent field in the cladding which was implemented to excite dye molecules for fluorescence microscopy image acquisition. The intensity in the image was proportional to the intensity of the exiting light at the location of the dye. The measured signal in this microscopy was a grey scale value, $\mathrm{G}$, in the image. The image data itself 
was a two dimensional grey scale matrix $\mathrm{G}(\mathrm{y} ; \mathrm{z})$, where the local information of a single pixel was recorded together with the grey scale value represented by a numerical value. The value was saved as an integer in the range of the chosen bit-depth of the image, discretizing the continuous set of possible signals. The grey scale value of a defined position $(\mathrm{y} ; \mathrm{z})$ in the sample was therefore subscripted by $\mathrm{G}(\mathrm{y} ; \mathrm{z})$. The following proportionality was given

$$
G(y ; z) \sim I_{f l}(y ; z)
$$

with $I_{f l}(y ; z)$ the intensity from the sample at position $(\mathrm{y} ; \mathrm{z})$. Furthermore, the signal is a function of the field intensity in x-direction, $I_{e v}(x)$, at the location of the fluorescent dye and was proportional to the dye concentration, $\rho(x, y)$, at the respective sample location. The dye concentration needed to be below its critical concentration where quenching occurred [33-35]. The evanescent field was approximated to be homogeneous in the $y$ - and z-direction in the region of the acquired images and attenuation due to waveguide losses in the $\sim 150 \mu \mathrm{m}$ field of view of the images was neglected. Thus we can write

$$
I_{f l}(y ; z)=I_{e v}(x) \cdot \rho(y, z)
$$

In the theoretical derivation of the planar waveguide, the mathematical description for the intensity of the evanescent field can be written as [36]

$$
I_{e v}(x)=D \cdot e^{(-2 \cdot q \cdot x)}
$$

with $\mathrm{D}$ being a constant that characterizes the starting intensity at the waveguide surface at $\mathrm{x}=0$, and $\mathrm{q}$ the decay parameter:

$$
q=\sqrt{\beta_{m}^{2}-\left(\frac{n_{1} \omega}{c}\right)^{2}}=\frac{2 \pi}{\lambda_{0}} \sqrt{n_{e f f, m}^{2}-n_{1}^{2}}
$$


with $\beta_{m}$ the propagation constant of the $\mathrm{m}^{\text {th }}$ waveguide mode, $n_{1}$ the refractive index of the cladding, $\omega$ the angular frequency and $\lambda_{0}$ the wavelength of the light wave propagating in the waveguide, respectively and $\mathrm{c}$ the speed of light.

Taking into account that the intensity of the evanescent field follows this expression, we can write the following proportionality

$$
G(y ; z) \sim I_{f l}(y ; z)=\rho(y ; z) \cdot I_{e v}(x)=\rho(y ; z) \cdot D \cdot e^{(-2 \cdot q \cdot x)}
$$

Assuming that the acquired grey scale value of a pixel is linearly related to the captured intensity, it follows

$$
G(y ; z)=\rho(y ; z) \cdot D \cdot e^{(-2 \cdot q \cdot x)}
$$

This equation links the grey scale value of a pixel $(y ; z)$ to the fluorescence event at the spatial position $(\mathrm{x} ; \mathrm{y} ; \mathrm{z})$. The right-hand side of the equation shows two unknowns: the density of the fluorescent dye $\rho(y ; z)$ and the location distance $\mathrm{x}$ of the dye from the waveguide surface. Thereby, the equation cannot be solved and a second grey scale matrix, a second image of the sample, had to be acquired under nearly identical conditions. For the second measurement, the same dye density was employed as before, only the field intensity was varied by selecting a different waveguide mode. Thus, the grey scale value, $G(y ; z)$, the constant $D$ and the decay parameter $q$ have to be subscripted as they were functions of the chosen guided mode. The two images acquired with two different waveguide modes therefore delivered the following equation system:

$$
\begin{array}{ll}
\text { I } & G_{1}(y ; z)=\rho(y ; z) \cdot D_{1} \cdot e^{\left(2 \cdot q_{1} \cdot x\right)} \\
\text { II } & G_{2}(y ; z)=\rho(y ; z) \cdot D_{2} \cdot e^{\left(2 \cdot q_{2} \cdot x\right)}
\end{array}
$$

where $\mathrm{G}_{1}(y ; z)$ and $\mathrm{G}_{2}(\mathrm{y} ; \mathrm{z})$ represented the pixel values in the first and the second image and the shapes of the evanescent fields are described by $\mathrm{D}_{1}, \mathrm{q}_{1}$ and $\mathrm{D}_{2}, \mathrm{q}_{2}$, respectively. 
Solving equation (9) for $\rho(y ; z)$, inserting the results into equation (8), rearranging, normalizing to the relative coupling efficiencies and solving for $\mathrm{x}$ leads to the dye location distance map $\mathrm{x}(\mathrm{z}$; y):

$$
x(z ; y)=\frac{\ln \left(\frac{G_{1, \text { norm }}(y ; z) \cdot D_{1}}{G_{2, \text { norm }}(y ; z) \cdot D_{2}}\right)}{2 \cdot\left(q_{2}-q_{1}\right)}
$$

A Matlab program was written to calculate the distance map $x(z ; y)$, implementing the field distribution simulation with the appropriate refractive index architecture from the used waveguide to acquire $q_{i}$ and $D_{i}$.

\section{Results}

\section{Dye distance map for staircase LB-film}

Mapping of the $13 \rightarrow 23$ layers-step in the LB-film sample (Fig.2) was performed with a waveguide of a thickness of $658 \pm 1 \mathrm{~nm}$ and a refractive index of $n_{W G}=1.841 \pm 0.001$ with its $\mathrm{TM}_{1}$ and $\mathrm{TM}_{2}$ mode. For the simulation, the evanescent fields (the half space above the waveguide) were assumed to be filled with stearic acid with a refractive index of 1.47 [28]. This step has a theoretical thickness increase of $25 \mathrm{~nm}$ for 10 perfectly deposited LB-monolayers [37]. The calculated dye distance map is depicted in Fig.4a as well as a sectional z-cut at y- position $=300$ pixel in Fig.4b. Clearly the LB-film step is visible in the distance map by an overall color change around $\mathrm{z}=600$ pixel. The map shows on the distance scale of $0-100 \mathrm{~nm}$ an inhomogeneous sample thickness in both parts of the sample. Having the decreasing transfer ratios and the perforated structure of the LB films in mind, the inhomogeneity of the film thicknesses was expected. The z-cut in Fig.4b confirms this inhomogeneity, however clearly depicts the step. 
Averaging the data from the z-cut delivered two heights, $58.3 \mathrm{~nm}(\mathrm{~S} . \mathrm{D}=8.2 \mathrm{~nm})$ and $38.3 \mathrm{~nm}$ $(\mathrm{S} . \mathrm{D}=10.1 \mathrm{~nm})$, providing a step height of $\sim 20 \mathrm{~nm}$, less than the expected $25 \mathrm{~nm}$ for the perfect layer system. This deviation from the ideal case was expected by the non-perfectly layered sample.

The absolute theoretical height of the 13 layer perfect film is $32.5 \mathrm{~nm}$ and $57.5 \mathrm{~nm}$ for the 23 layer film. The measured average heights for both parts of the step are slightly overestimated, however lying well within the sample noise of $\sim \pm 10 \mathrm{~nm}$ due to the non-perfect LB transfer. This discrepancy is directly due to the implementation of an imprecise refractive index for the stearic acid in the evanescent field simulations, as the refractive index was only an assumption from reference [28] and taken to be homogeneous along the entire LB film thickness which is obviously not the case.

To test the reproducibility, one stepped LB-film sample was used to take images on a particularly chosen step several times by removing it from the microscope, storing it for a day or two and then realign it for imaging. In the z-scan analysis, all experiments delivered the same averaged film thicknesses and step height within the experimental errors.

\section{Dye distance map for fixed osteoblasts}

A waveguide with a thickness of $651 \pm 2 \mathrm{~nm}$ and a refractive index of $\mathrm{n}=1.840 \pm 0.001$ was used for mapping the distances of the dye located in the plasma membrane of fixed osteoblasts. The volume above the waveguide was assumed to be water with a refractive index of 1.33 for simulating the evanescent fields. Images taken with $\mathrm{TM}_{1}$ and $\mathrm{TM}_{2}$ modes were used to calculate the dye distance map. Fig.5 shows as an example a color code distance map of four osteoblasts and in the inset an overexposed WEFF image of the same field of view. The WEFF image depicts the four osteoblasts well spread and indicating the nuclei and some cell extensions. The dye 
distance map depicts lower distance-colors (blue to yellow) in the area of the cells from $\sim 130 \mathrm{~nm}$ to close to $0 \mathrm{~nm}$. In the unoccupied area, the unstained medium, where the raw data do not show fluorescence intensities, only noise is present which is depicted as x-distances $>130 \mathrm{~nm}$ (mixed yellow and red pixels). The method shows no-sample area, areas where no fluorescence signals were emitted, as high $\mathrm{x}$-distance areas close to and beyond the penetration depth of the evanescent field. (The signal is so weak that it must come from far away.) The differences between both images (which were used to calculate the distance map) outside the cells are caused by variations in the background noise. These noise variations lead to an image noise-level, here of mixed yellow and red pixels. In addition, isolated spots in the no-sample area (outside the cells) are visible in very dark blue. These spots are correlated to un-physical distance values below zero caused by microscopic damage to the waveguide. These un-physical distances should always be omitted in image interpretation.

All four osteoblasts can be found in the distance map and show cell outlines similar to the cells depicted in the overexposed WEFF image. However, the lamellipodia and the thinly spread cell body are even clearer in the distance map. In Fig.5, the distance map does not depict any information about the nuclei. Not the entire cell body reached down very close to the surface, as expected. At some of the cells' outer lines and at some extreme tips of the spread cells, small regions - only a few pixels in diameter - were found with distances of $\sim 10-25 \mathrm{~nm}$, typical of a FA $[4,16,17]$. Twice line like accumulations of dense FAs are found (blue lines with distances around $10-25 \mathrm{~nm}$ ). Between the FAs, there are regions in lighter blue depicting distances around $40-50 \mathrm{~nm}$ as well as greenish areas depicting distances around $70-80 \mathrm{~nm}$. Lamellipodia of the cells, which are very faintly seen in the WEFF images, are clearly visible in the distance map as 
thin spikes with a blue (possible FAs or PCs) or green (possible EMCs) center and green-yellow surroundings.

Fig.6 depicts one well spread osteoblast in overexposed WEFF and false color distance map imaging. Two z-cuts through the distance map have been made: one randomly through the cell, (Fig.6c) and one through an area including the small distances of the cell (Fig.6d) (see Fig.6b for precise cut locations). The area outside the cell is nearly homogeneously dark red colored (distances $>90 \mathrm{~nm}$ ) showing that there is no sample. The existing noise level in the no-sample regions is clearly depicted in the z-cut data; it is the noisy data at an average distance of $\sim 90 \mathrm{~nm}$ on both sides of the cell. The cell itself is shown by the depressions in the z-cuts. The spreading of the cell is excellently depicted by the distance map.

The cell is attached at all extreme spreading points, however not necessarily as FAs since, distances above $40 \mathrm{~nm}$ and up to $50 \mathrm{~nm}$, possible CCs, are found. In the center of the cell, FAs are present. The cell's plasma membrane seems to bend away from the waveguide surface close to the $80 \mathrm{~nm}$ bright red area where the nucleus is located marking its location in the distance map.

The z-cuts show the position of the plasma membrane/dye location along the cut line in nm. For the random cut 'c' (Fig.6c), three "small” distances in the order of $\sim 55 \mathrm{~nm}$ are found, as well as a couple of more bends towards the substratum with distances of $\sim 62-67 \mathrm{~nm}$. The maximum heights of the plasma membrane from the waveguide surfaces between the bends towards the substratum are found to be between 62 and $75 \mathrm{~nm}$. These bends towards the substratum might be CCs or ECMs.

In the z-cut ' $d$ ' through the small distance adhesions (Fig.6d) one FA at $18 \mathrm{~nm}$ is found as well as CCs with distances of $25-35 \mathrm{~nm}$. The maximum heights of the plasma membrane from the waveguide surfaces in this case are 37 and $45 \mathrm{~nm}$. The bending of the membrane towards the 
cytoplasm between these adhesions points is clearly seen. It is unclear at this point whether these bends towards the cytoplasm in the membrane with distances of $37-67 \mathrm{~nm}$ represent EMCs or not. However, the relative straight lines between the "maxima and minima" in the distance curve bear more resemblance to a stretched rubber band and not to a patchy assembly of individual EMCs. One needs to keep in mind that the surface tension of the plasma membrane tries to minimize the surface area, trying to force the cell into a spherical shape. The adhesions are obvious biological disruptions of the physical effect of surface tension. Therefore it seems likely that the ends towards the cytoplasm are not positions of EMCs.

Fig.7 depicts one cell from Fig.5 in more detail - the osteoblast on the far right with its bright field image, dye distance map, a z-cut through a couple of near distance adhesion points and a 3D-contour plot. In the contour plot, the closest points are represented by blue "mountain tops".

The z-cut in Fig. 7 shows three areas with distances of $\sim 8, \sim 18$ and $\sim 19 \mathrm{~nm}$. As the cut is run centrally to the closest distances, these are probably FAs. An additional dip with a distance of $\sim 41 \mathrm{~nm}$ is found at z-position $\sim 168 \mu \mathrm{m}$. This represents a CC as the area around it shown in Fig. $7 \mathrm{a}$ shows very similar colors. The various distance dips between 50 and $90 \mathrm{~nm}$ are plasma membrane segments bending down to the substrate to this particular distance by a nearby adhesion as seen by comparing to Fig.7a. Between these "adhesions", the detailed z-cut depicts the bending of the plasma membrane towards the cytoplasm with maximum distances $90-100 \mathrm{~nm}$, again describable mostly as straight lines. The contour plot in Fig.7d gives an overall impression of the 3D- plasma membrane location with respect to the substratum along the entire cell. The blue "mountain tops" represent the closest adhesion points and are found within the cell but also along the cell extensions, as expected. The Supplemental Material shows the 3D-contour plot rotating for further detail. 
All the above images were obtained from fixed cells. Live cell distance determination along the entire cell area was not possible, since image acquisition took too long with the camera currently used and the non-automated mode coupling and changing procedures. Too much time passed between taking two images so that cells had moved, in particular at the margins and therefore time-lapse distance mapping was not possible. A decrease in imaging time and an automated mode coupling system will allow time lapse dye distance mapping in the future so that dynamics in the adhesions can be monitored in real time.

Also for the osteoblast samples, repeated imaging over several days was performed on the fixed samples. For a couple of picked adhesion sites the distances were found to be reproducible within the experimental error. Even the living cells delivered reproducible data (not shown) on focal adhesions which did not move during the imaging time even when imaged over a couple of hours. Areas showing cell movement in the live cell culture were of course not reproducible.

\section{Discussion}

Multimode WEFF microscopy conducted with well characterized waveguides and in combination with simulation of the evanescent fields generated by the waveguide modes makes it possible to calculate a dye distance map with a simple mathematical procedure. Z-cuts and 3Dcontour plots are possible, which show a relief map of the plasma membrane along the substratum. As this is refraction limited microscopy, the lateral resolution is in order of $1 \mu \mathrm{m}$, whereas the distance resolution lies is the nm range. However, various issues have to be taken into account for error analysis in the distance determinations. An excellent discussion on refractive index issues in TIRF and IRM is found in Burmeister [3]. WEFF microscopy involves similar problems. 
In addition, the fundamental assumption of an exponentially decaying field and fluorescent signals, which follow this function linearly, should be considered. A single fluorescent molecule can be treated as an electric dipole that is excited by a photon. For the dipole near a dielectric interface, it is however possible to emit not only into a propagating but also into an evanescent wave. This evanescent wave is limited to the interface and cannot be acquired by common microscopic optical components. Thus, the theoretical fluorescence detection behaviour is altered from a pure exponential decay. This phenomenon was observed on fluorescent granules in the evanescent field of a TIRF microscope for distances less than $150 \mathrm{~nm}$ from the interface [38]. A second effect is able to reduce the collected fluorescence intensity near the surface. Radiation from the dye molecules, which is emitted towards the interface, can be reflected and causing interference with the directly radiated wave of the dipole. Leutenegger and Lasser observed that the dissipated power is traceable for distances of less than $\lambda / 5$ [39]. To increase the total amount of collected fluorescence, both groups suggest the use of objectives with high numerical aperture, e.g. 1.45. These near-surface effects are neglected in most publications on distance measurement by exploiting the evanescent field. Since in all experiments the fluorescence dye molecules were located further away than $10 \mathrm{~nm}$ from the surface, and the two modes are similarly prone to this problem, this error source was ignored here as well. Nevertheless, this issue should be kept in mind for future improvements.

While mapping the LB film step, it became obvious that a precise knowledge of the refractive index of the material directly adjacent to the waveguide is of high importance for calculating true distances. Even the homogeneity or inhomogeneity of the refractive index along the distance direction plays a major role. In addition, in thin films deposited with LB technology a high anisotropy in the layer structure is present. The molecules are oriented preferentially with 
their long axis perpendicular to the substrate. Imaging with TM modes means probing these layers with a polarization direction oriented primarily along the main molecular axis of the stearic acid where the transition dipole moment is lying, and therefore, the refractive index is higher in comparison to imaging with the perpendicular direction, TE modes. The intensity at the interface, $\mathrm{D}$, as well as the decay parameter, q, of the evanescent field, which are both delivered by the waveguide simulation via ATSOS, are dependent on the refractive index and its structure of the cladding on the waveguide.

Taking this refractive index uncertainty into account, both individual heights of the step as well as the step height, a relative distance, were determined very well within the errors due to the non-perfect sample and in comparison to the theoretical thickness of a perfect LB film. The slight overestimation of the absolute heights is a pure refractive index issue.

For the cell mapping, the data analysis was carried out by assuming that no molecules from the culture medium were adsorbed onto the waveguide - the cladding refractive index was simulated as water with $\mathrm{n}=1.33$, as in TIRF and IRM data analysis [3]. Right at the adhesion sites, the true refractive index is definitely different from that of water as the integrins are present at these locations with a refractive index larger than that of water. They are also oriented in a particular fashion leading to a highly anisotropic refractive index structure. This will locally deform the evanescent field, again depending on the chosen polarization (TE or TM), changing D and q locally. However, as the individual adhesion is smaller than the diffraction limit of the optical system [19] the distance right at the location of the adhesion, the individual integrin molecule cannot be measured or calculated. Obviously, the calculated distance where the adhesion is located is false as the refractive index in the cladding at this location is assumed too low. On the other hand, right at the position where the integrin is located, no dye molecule can be located. In addition, 
the distance calculation is done per pixel which is much larger than an individual integrin adhesion and therefore the pixel "smears out" to areas to the sides of the adhesion to where dyes are present in the membrane (and deliver a signal) and where water is the filling material to the substratum. The calculated distance is an average of the dye distances in the pixel and cannot be better than the diffraction limit of the method which is $<1 \mu \mathrm{m}^{2}$. Therefore only minor errors are produced by omitting the enhanced integrin refractive index in the adhesions within one pixel. All pixels surrounding the adhesion pixel are treated precisely. The difference in the distance value calculated from the pixel with the adhesion and the pixels from the nearest surrounding is so low that within the noise of the method it does not show up in the data. Therefore, the data shown in the distance maps (Figs.4-7) are reliable, of course within the experimental error.

The most serious sources of error in the distance mapping procedure are from determining the coupled intensity, which is necessary for normalizing the WEFF images and from possible laser power fluctuations. For the system implemented here, about 3\% errors are produced by these two error sources. Simulations, taking a deviation of 5\% into account, have resulted in a distance error of $\pm 10 \mathrm{~nm}$. The waveguide characterization parameters that are needed for the calculation of $\mathrm{D}$ and $\mathrm{q}$ and therefore in distance calculation, such as thickness of the waveguide, refractive index of the waveguide, refractive index of the substrate and excitation wavelength, can be determined precisely enough to neglect their influence on the uncertainty of the results. The errors in Fig.5-7 are therefore very similar to the errors in TIRF, in the order of $\pm 7 \mathrm{~nm}[3]$.

In principle, the method presented here can be expanded by using more than two modes to calculate the distance map. In this case, the system will be over-determined and one would be able theoretically to decrease the errors. In practice, the errors would be still in the order of a few $\mathrm{nm}$ as the uncertainties of the experimental parameters will not be decreased; the coupling efficiency 
measurement needs to be much more precise than as currently done. A multimode approach will help in gaining a more precise knowledge on all involved refractive indices.

In conclusion, WEFF mapping microscopy is able to display quantitatively ultra-thin steps in thin film samples as well as depict the distances between the plasma membrane of a cell and its substratum along an entire cell. The various adhesion forms can be identified as well as the bending of the membrane between adhesions. Comparing the values of the closest plasma membrane distances with previous reported distances of osteoblasts [15,20-22], we seldom find a focal adhesion but often contacts with larger distances that are typical for cells on glass surfaces [40].

Even in highly sophisticated phase-sensitive novel IRM methods, such as laser feedback interference microscopy, where it is possible to separately determine the variation in index of refraction (fringe visibility) from the effect of variations in the distance to the ventral membrane surface, a more precise distance determination is difficult [19]. However, by automating the microscope in such a way that it quickly shifts between the two modes, time lapse distance mapping should be possible. This will allow investigators to quantitatively study the kinetics of adhesion in live cells. 


\section{Acknowledgments}

These studies were supported in part by the Canadian Institutes of Health Research (grant number

102542), NSERC and the RheinMainUniversity of Applied Sciences by a travel grant. We thank Elisabeth Pruski for all the help with the cells and the Western Nanofabriaction Facility to host our LB trough. 


\section{References:}

[1] X.F. Niu, Y.L. Wang, Y.L. Luo, J. Xin, and Y.G. Li, Journal of Materials Science \& Technology 21, $571-576$ (2005).

[2] H. Storrie, M.O. Guler, S.N. Abu-Amara, T. Volberg, M. Rao, B. Geiger, and S.I. Stupp, Biomaterials 28, 4608 - 4618 (2007).

[3] J.S. Burmeister, L.A. Olivier, W.M. Reichert, and G.A. Truskey, Biomaterials 19, 307 325 (1998)

[4] W.T. Chen, and S.J. Singer, Journal of Cell Biology 95, 205 - 222 (1982).

[5] J.S. Burmeister, G.A. Truskey, and W.M. Reichert, Journal of Microscopy-Oxford 173, 39 $-51(1994)$.

[6] K.F. Giebel, C. Bechinger, S. Herminghaus, M. Riedel, P. Leiderer, U. Weiland, and M. Bastmeyer, Biophysical Journal 76, 509 - 516 (1999).

[7] H. Verschueren, J Cell Sci 75, 279 - 301 (1985).

[8] D. Braun, and P. Fromherz, Applied Physics A-Materials Science \& Processing 65, 341 348 (1997).

[9] E. Atilgan, and B. Ovryn, Current Pharmaceutical Biotechnology 10, 508 - 514 (2009).

[10] M. Abercrombie, J.E. Heaysman, and S.M. Pegrum,. Exp. Cell Res. 67, 359 - 367 (1971).

[11] H.M. Grandin, B. Städler, M. Textor, and J. Vörös, Biosensors \& Bioelectronics 21, 1476 $-1482(2006)$

[12] B. Agnarsson, S. Ingthorsson, T. Gudjonsson, and K. Leosson, Optics Express 17, 50755082 (2009).

[13] R. Horvath, H.C. Pedersen, N. Skivesen, D. Selmeczi, and N.B. Larsen, Applied Physics Letters 86, 071101 (2005). 
[14] A. Hassanzadeh, M. Nitsche, S. Mittler, S.J. Armstrong, J. Dixon, and U. Langbein, Applied Physics Letters 92, 233503 (2008).

[15] A. Hassanzadeh, S. Armstrong, S.J. Dixon, and S. Mittler, Applied Physics Letters 94, 033503 (2009).

[16] A. Hassanzadeh, M. Nitsche, S. Armstrong, N. Nabavi, R. Harrison, S.J. Dixon, U. Langbein, and S. Mittler, Journal of Biomedical Optics 15, 036018-1 - 036018-7 (2010).

[17] C.O. Arregui, S. Carbonetto, and L. McKerracher, The Journal of Neuroscience 14, 6967 -6977 (1994).

[18] N. Tawil, E. Wilson, and S. Carbonetto, Journal of Cell Biology 120, 261 - 271 (1993).

[19] E. Atilgan, and B. Ovryn, Current Pharmaceutical Biotechnology 10, $508-514$ (2009).

[20] G. Schneider, and K. Buridge, Experimental Cell Research 214, 264-269 (1994).

[21] K. Anselme, Biomaterials 21, 667-681 (1999).

[22] A. Diener, B. Nebe, F.Lüthen, P. Becker,U.Beck, H.G. Neumann, J. Rychly, Biomaterials 26, 383-392 (2005).

[23] A. Hasanzadeh and S. Mittler, Journal of Biomedical Optics 16, 046022 (2011).

[24] M Xu, R. Moshrefzadeh, U.J. Gibson, G.I. Stegeman, and C.T. Seaton, Applied Optics 24, $3155-3161(1985)$.

[25] E. Ertorer, F. Vasefi, J. Keshwah, M. Najiminaini, C. Halfpap, U. Langbein, J.J.I. Carson, D.W. Hamilton, and S. Mittler, Biomedical Optics 18, 035002 (2013).

[26] T.M. Lee, S. Mittler-Neher, D. Neher, G.I. Stegeman, C. Roux, M. Leclerc, J. Martin, S.I. Najafi, Optical Materials 1, 65 - 70 (1992). 
[27] ATSOS - Analysis Tool for Stratified Optical Structures, DOOS, Übelbergweg 1, 99891 Tabarz, Germany, http://doos.de/

[28] A.M. Brzozowska, M.H.G. Diuts, and F. Mugele, Colloids and Surfaces A: Physicochem. Eng. Aspects 407, $38-48$ (2012).

[29] M.C. Petty, Langmuir-Blodgett Films: An Introduction, Cambridge University Press (1996).

[30] L.L. Lanier and N.L. Warner, Journal of Immunological Methods 47, 25-30 (1981).

[31] J.W. Smit, C.J.L.M. Meijer, F. Decay, and T. M. Feltkamp, Journal of Immunological Methods 6, 93-98 (1974).

[32] J.-W. Su, W.-C. Hsu, J.-W. Tjiu, C.-P. Chiang, C.-W. Huang, and K.-B. Sunga, Journal of Biomedical Optics 19, 075007 (2014).

[33] J.R. Silvius, Annual Review of Biophysics and Biomolecular Structure 21, $323-348$ (1992).

[34] A. Hassanzadeh, H. Kan Ma, S.J. Dixon, and S. Mittler, Biomedical Optics 17, 076025 17 (2012).

[35] F. Fleißner, Master Thesis, Distance determination of fluorescence dye via WEFF Microscopy. Hochschule RheinMain, Wiesbaden/Rüsselsheim (2013).

[36] W. Karthe, and R. Müller, Integrierte Optik, Akademische Verlagsgesellschaft, Leipzig (1991).

[37] W.A. Barlow, (ed.) Langmuir-Blodgett Films, Elsevier (1980).

[38] A. Rohrbach, Biophysical Journal 78, 2641 - 2654 (2000).

[39] M. Leutenegger, and T. Lasser, Optics Express 16, 8519 - 8531 (2008). 
[40] D. K. Knight, R. Stutchbury, Daniel Imruck, C. Halfpap, S. Lin, U. Langbein, E. R. Gillies, S. Mittler and K. Mequanint, Applied Materials and Interfaces 4, 1303-1312 (2012).

\section{Figure Captions}


Fig.1 Schema of WEFF microscopy.

Fig.2 a) Scheme of LB-film staircase sample on the waveguide. b) SEM image of the transition region from 13 to 23 layers with the final DiO layer on top. The individual lines marked by arrows represent the displacement between the depositions of double layers within the desired step due to the evaporation of the water subphase in the trough during the drying period after each stroke. The LB-films show irregular holes explaining the low transfer ratio. The scale bar represents $300 \mathrm{~nm}$.

Fig.3 Scheme of two different evanescent fields of two waveguide modes. At a particular distance $\mathrm{x}$ from the waveguide surface $(\mathrm{x}=0)$, the fluorescence intensity of a dye molecule located at distance $\mathrm{x}$ is proportional to the local intensity $\mathrm{I}(\mathrm{x})$ being different for different modes.

Fig. 4 a) Dye distance map of the $13 \rightarrow 23$ layers-step in the LB-film sample taken at a laser power of $8.7 \mathrm{~mW}$ at $488 \mathrm{~nm}$, with a 40x objective, an exposure time of $180 \mathrm{~s}$, gain 1, and $\mathrm{TM}_{1} / \mathrm{TM}_{2}$ modes. The scale bar represents $50 \mu \mathrm{m}$. b) Sectional z-cut at y-position $=300$ pixel and fitted to constant height.

Fig.5 False color representation of a dye distance map with four osteoblasts. The inset represents an overexposed WEFF image of the same field of view. Osteoblasts appear at dye/plasma 
membrane distances between $\sim 75$ to $110 \mathrm{~nm}$. Dye locations very close to the surface, adhesion areas, are labeled blue. Both scale bars represent $50 \mu \mathrm{m}$. The color bar shows a range from - $50 \mathrm{~nm}$ to $200 \mathrm{~nm}$. One pixel is equal to $(0.266 \mu \mathrm{m})^{2}$.

Fig.6 Single osteoblast. a) Overexposed WEFF image, b) false color representation of the dye distance map, c) z-cut through cell at random position 'c' in part b) and d) z-cut through cell at smallest distance locations at position ' $d$ ' in $b$ ). The cuts in b) from bottom to top are represented in c) and d) from left to right.

Fig.7 Detailed view of one osteoblast: a) distance map, b) bright field image, and c) z-cut and d) a 3D-contour plot showing attachments as blue "mountains'. The scale bars represent $50 \mu \mathrm{m}$. The cut in a) from bottom to top is represented in c) from left to right.

\section{Figures}


FIG.1

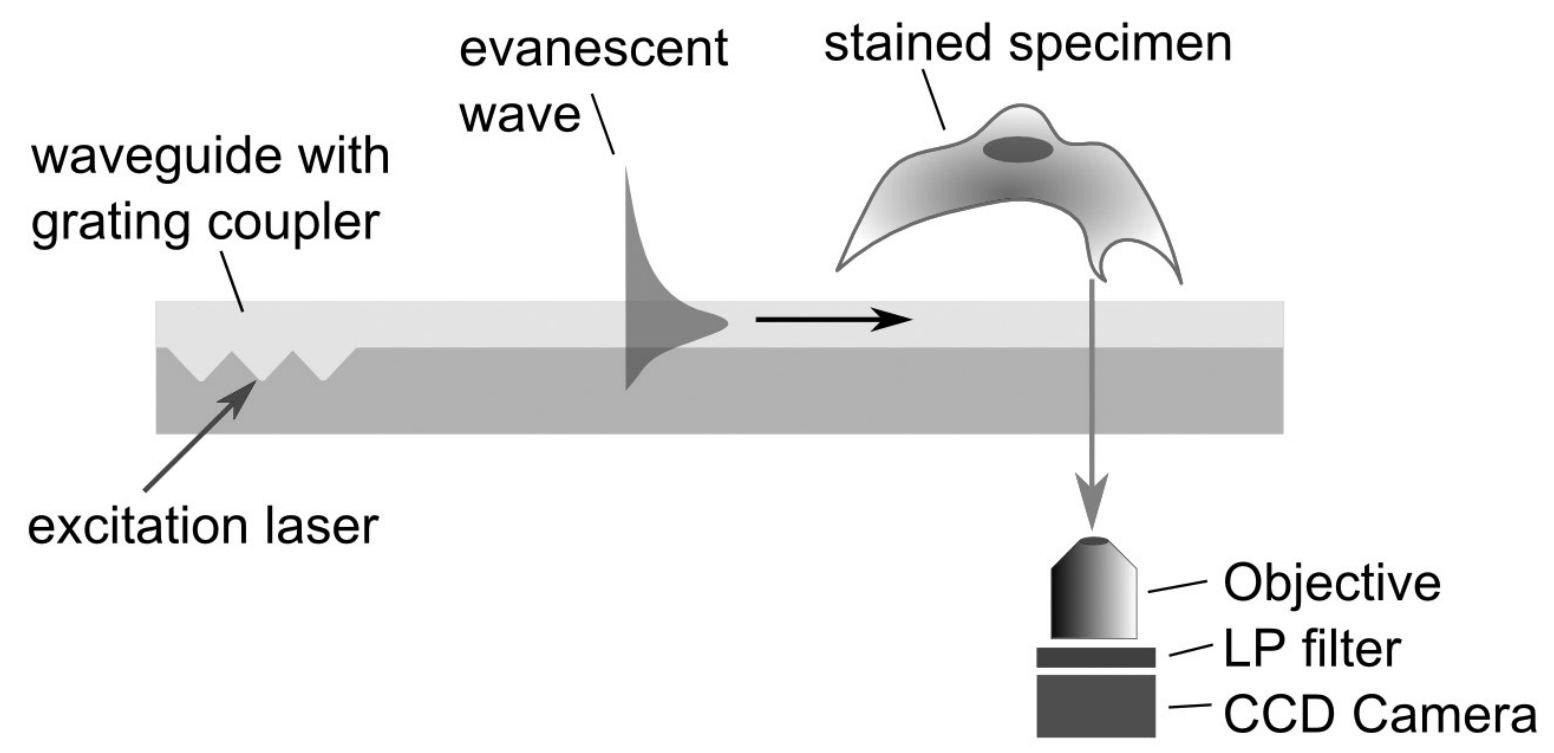

FIG.2a

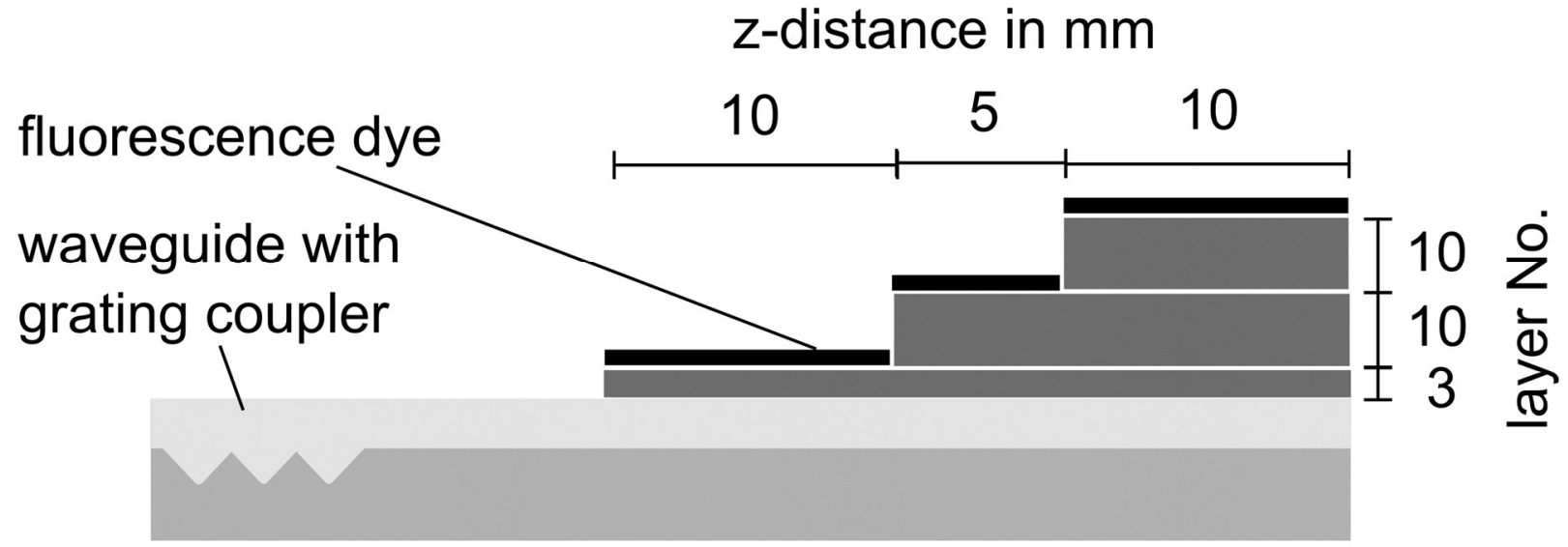

FIG.2b 


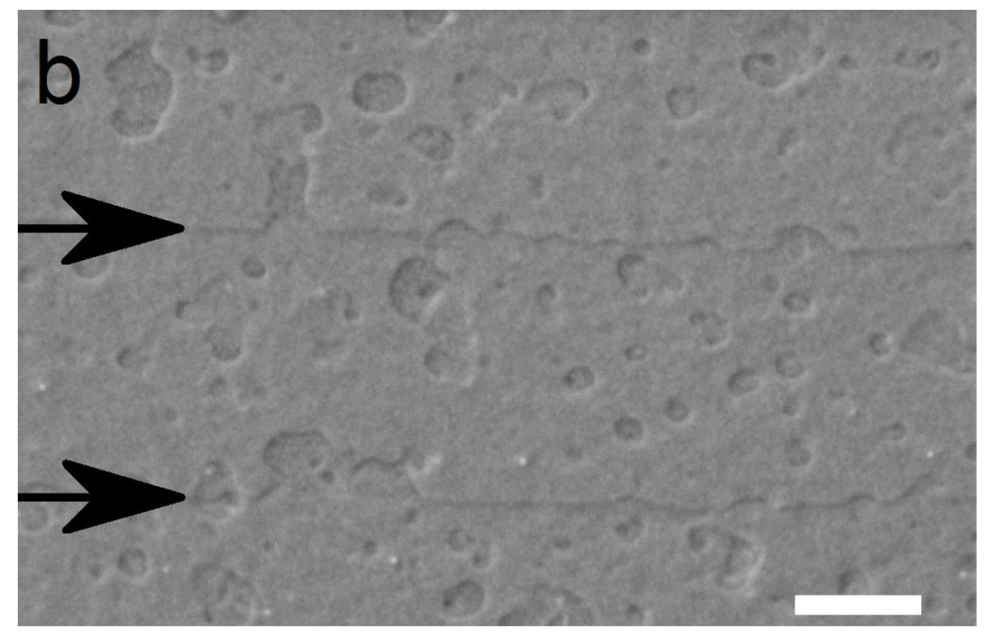

FIG.3

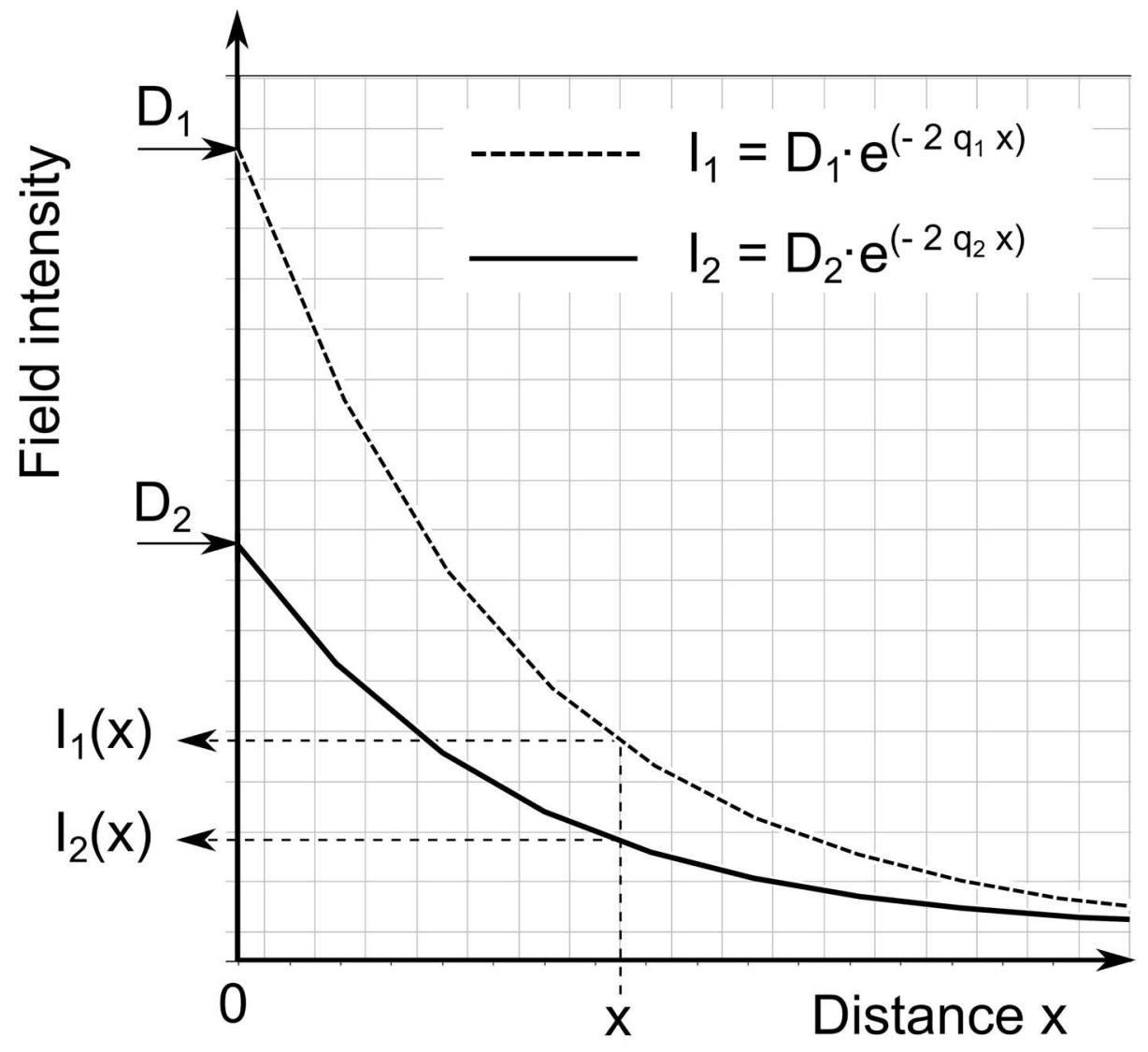

FIG.4a 


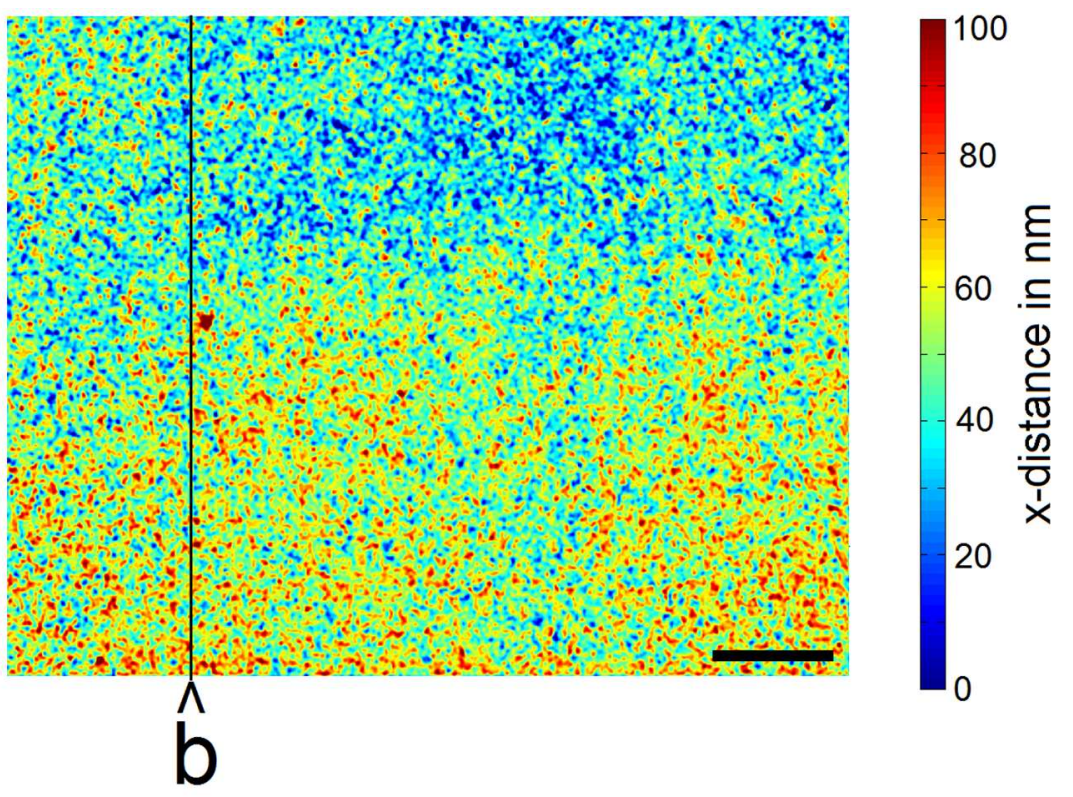

FIG.4b

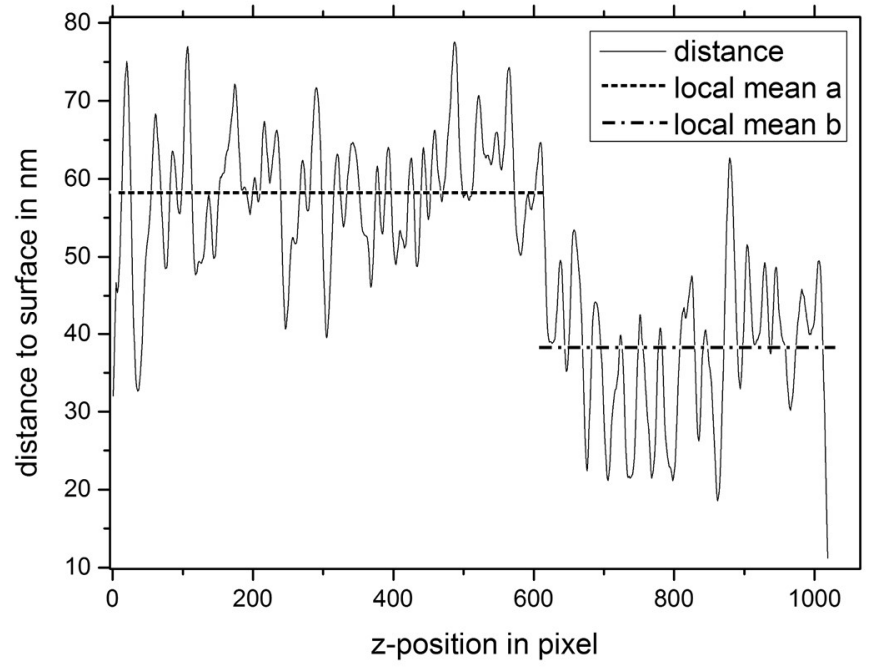

FIG.5 


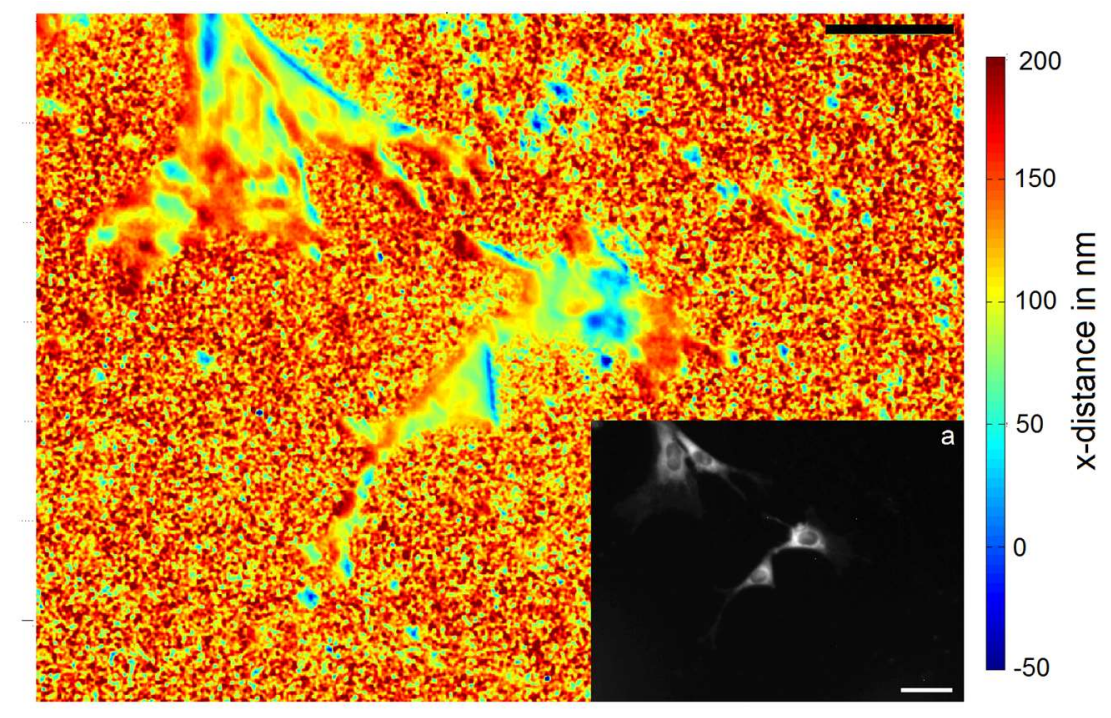

FIG.6
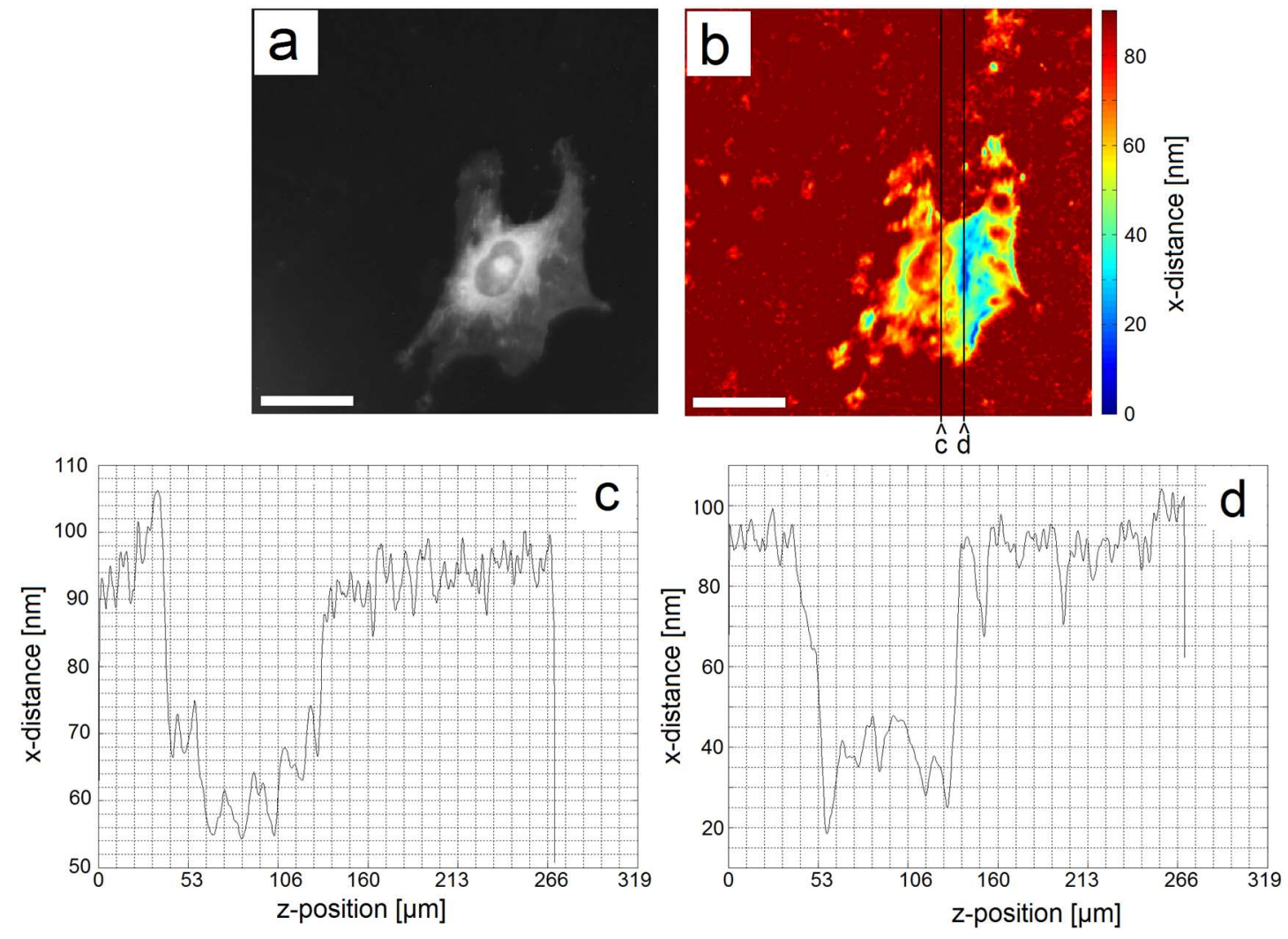

FIG. 7 

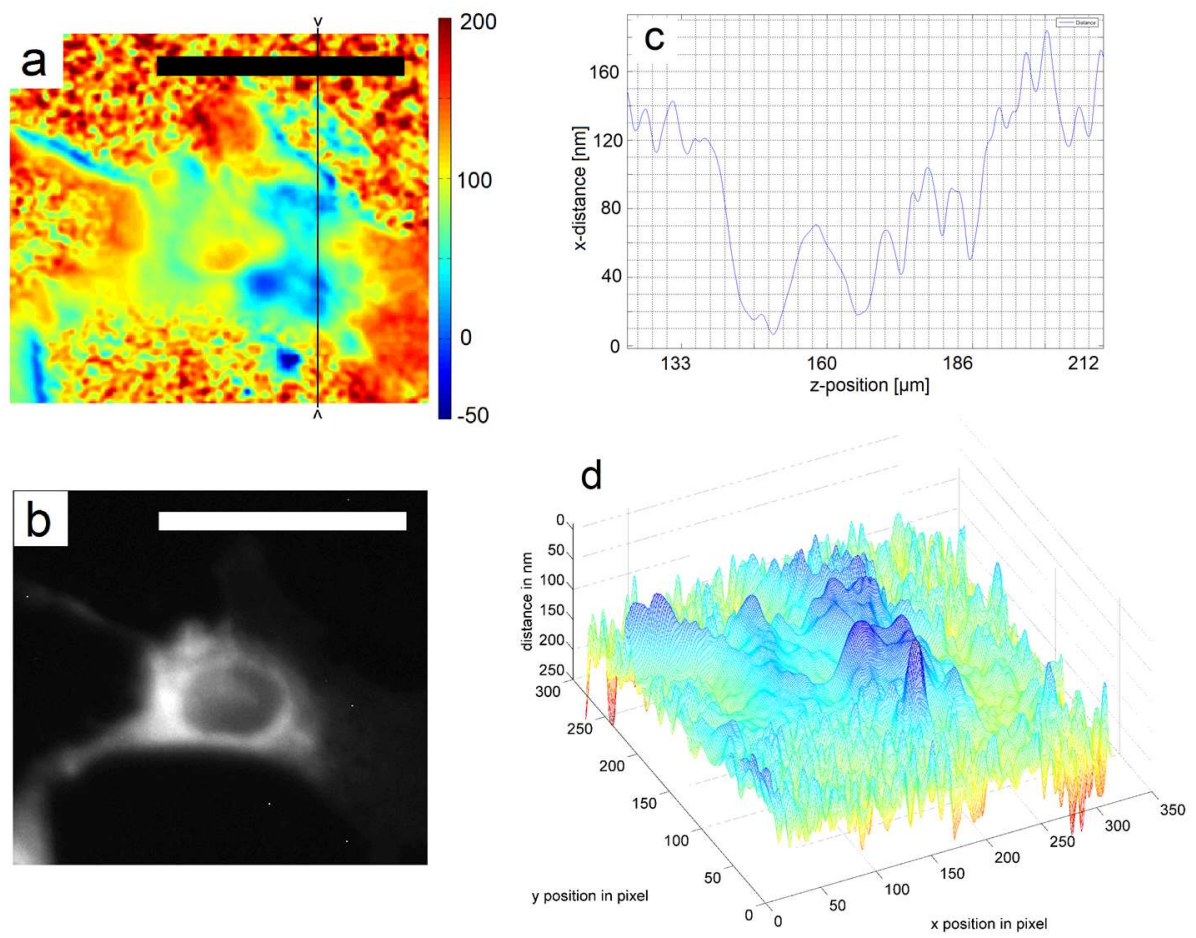